\title{
MPEG-2 Transcoding Method for Reducing Re-quantization Noise Based on a Two-tiered Quantizer Matrix
}

\author{
（2 段型量子化マトリックスを用いた量子化雑音の少ない MPEG-2トランスコード手法）
}

Takeshi Yoshitome $^{\dagger}$, Jiro Naganuma ${ }^{\dagger \dagger}$, and Yoshiyuki Yashima (member) ${ }^{\dagger \dagger}$

\begin{abstract}
We developed an MPEG-2 transcoding method on the basis of a two-tiered quantizer matrix that reduces re-quantization noise. The proposed method changes the quantization matrix to decrease the re-quantization noise, instead of changing the value of the quantization parameters as conventional methods do. Using this two-tiered matrix makes it possible to reduce re-quantization noise in the high-frequency domain. The main feature of the proposed method is that it does not cause any differences in image quality between adjacent macroblocks, which the conventional method does. Experimental results show that our proposed method produces transcoding scores of structural similarity (SSIM) and video quality model (VQM) that are 0.044 and 0.0938 , respectively, better than those of the best conventional method. The proposed method always produced superior SSIM and VQM scores when the bit reduction ratio was smaller than 0.1015 for all the tested sequences.
\end{abstract}

Key words: re-encoding, transcoding, MPEG-2, quantization noise

\section{Introduction}

Recently, many kinds of video services have begun to provide compressed video content to consumers. High-quality and real-time transcoding technologies are needed for such visual applications, and many approaches to high performance transcoding have been studied $^{1)-4)}$. The most important factor in transcoding is to reduce re-quantization noise mixed during the transcoding procedure. It is well known that repeating all decisions made during the first encoding procedure is an effective method for reducing re-quantization noise during the transcoding procedure ${ }^{5) 6(8)}$. These decisions include those made on characteristics, such as picture type, macroblock type, motion vector, DCT type, and quantization parameters. Transcoders that use these characteristics are suitable for real-time video transcoding because they do not require the computation time normally needed for such factors as motion search and macroblock mode selection. However, this does not en-

Received February 23, 2009; Revised May 8, 2009; Accepted July 14, 2009

$\dagger$ NTT Cyber Space Laboratories, NTT Corporation

(1-1, Hikarinooka, Yokosuka, Kanagawa, 238-0042, Japan)

$\dagger \dagger$ NTT Electronics Corporation

(1841-1, Tsuruma, Machida, Tokyo, 194-0004, Japan)

$\dagger \dagger \dagger$ Faculty of Information and Computer Science, Chiba Institute of Technology

(2-17-1, Tsudanuma, Narashino, Chiba, 275-0016, Japan) able bit reduction because all decisions made, including those concerning quantization parameters, are repeated during the transcoding procedure.

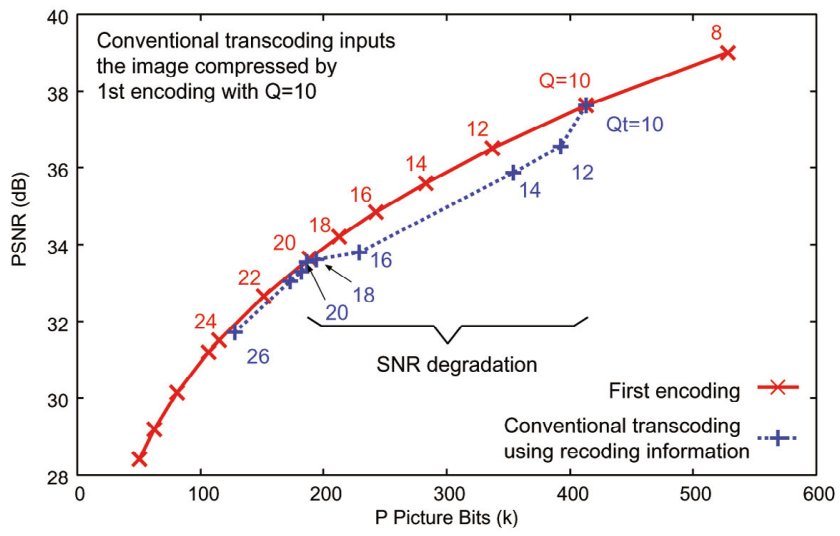

Fig. 1 PSNR example of encoding and transcoding in MPEG-2.

An example of re-quantization noise in the transcoding procedure is shown in Fig. 1, which illustrates the relationship between picture bits and PSNR as it relates to inter-frame MPEG-2 encoding. The solid line indicates the dependence of peak signal-to-noise ratio (PSNR) on the number of picture bits in the first encoding, and $Q$ is the quantization parameter of the first encoding. The short dotted line indicates the PSNR of conventional transcoding, which uses all the informa- 
tion of the first encoding procedure except the quantization parameter. The input image of the transcoder is already compressed by the first encoding, where the first cncoder's quantization parametcr, $Q$, is cqual to 10. $Q t$ denotes the quantization parameter of conventional transcoding. The PSNR of the first encoding, where $Q=10$, is almost the same as that of conventional transcoding, where $Q t=10$. This means that no additional noise is caused by the transcoder when the transcoding parameter value is equal to the first encoding parameter. This also shows that no additional noise is incurred in conventional transcoding when $Q t$ is equal to double $Q$. If the $Q / Q t$ ratio is between 0.5 and 1.0 , there is a clear degradation in the PSNR characteristic. In the following section, we will describe the reason PSNR degradation occurs in conventional transcoders.

\section{Re-quantization noise in transcoder}

In this section, we briefly review quantization and de-quantization procedures to understand the requantization noise generated by transcoders.

The quantization and de-quantization procedures for intra macroblocks in MPEG-2 TM5 ${ }^{13)}$ are shown in Eq. (1) and (2).

$$
\begin{gathered}
k_{i, j}=\operatorname{int}\left(\frac{32 x_{i, j}+\operatorname{sign}\left(x_{i, j}\right) \cdot Q \cdot(p / q) \cdot W I_{i, j}}{2 \cdot Q \cdot W I_{i, j}}\right)(1) \\
\hat{x}_{i, j}=\operatorname{int}\left(\frac{k_{i, j} \cdot Q \cdot W I_{i, j}}{16}\right) \\
\text { where } \operatorname{sign}\left(x_{i, j}\right)= \begin{cases}1 & x_{i, j}>0 \\
0 & x_{i, j}=0 \\
-1 & x_{i, j}<0\end{cases} \\
p=3, \quad q=4
\end{gathered}
$$

The quantization and de-quantization procedures for inter macroblocks, are shown in Eq. (3) and (4).

$$
\begin{aligned}
& k_{i, j}=\operatorname{int}\left(\frac{16 x_{i, j}}{Q \cdot W N_{i, j}}\right) \\
& \hat{x}_{i, j}=\operatorname{int}\left(\frac{\left(\left(2 \cdot k_{i, j}+\operatorname{sign}\left(k_{i, j}\right)\right) \cdot Q \cdot W N_{i, j}\right)}{32}\right)
\end{aligned}
$$

where $x_{i, j}, k_{i, j}, W I_{i, j}, W N_{i, j}$, and $Q$ denote the $(i, j) t h$ coefficient of the DCT output, the quantized value of the DCT output, the intra quantization matrix, the inter quantization matrix, and the Qstep value, respectively. The quantization noise, mixed in the CODEC, is the difference between $x_{i, j}$ and $\hat{x}_{i, j}$.

The block diagram of a CODEC chain that consists of two decoders and two encoders is shown in Fig. 2. Encoder \#1 compresses the input video signals and generates bitstream \#1 for the transcoder. The transcoder, which consists of decoder \#1 and encoder \#2, decodes

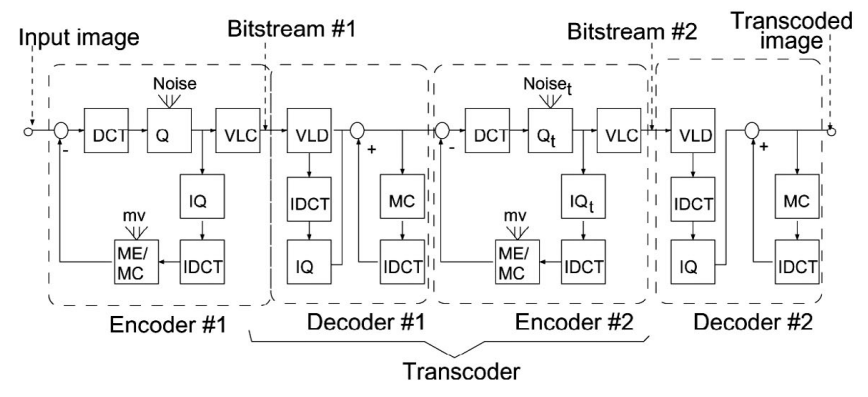

Fig. 2 CODEC chain with transcoder.

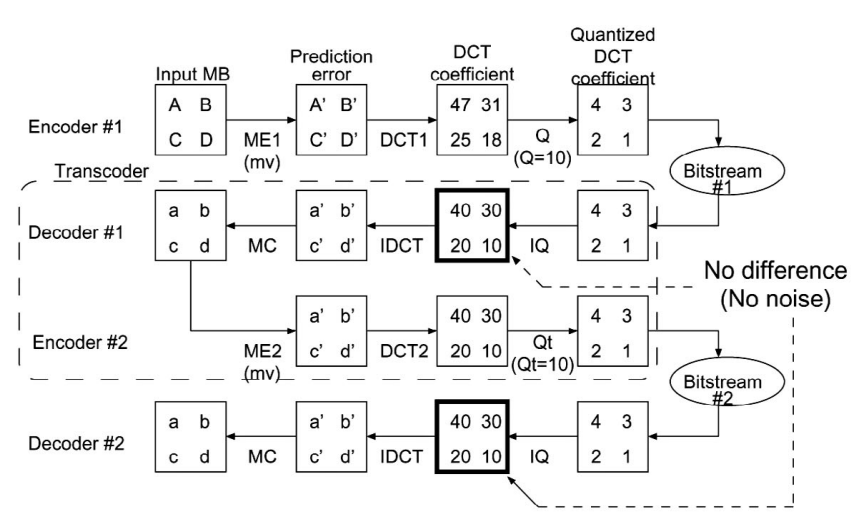

Fig. 3 Dataflow of CODEC chain $(\mathrm{Q}=\mathrm{Qt}=10)$.

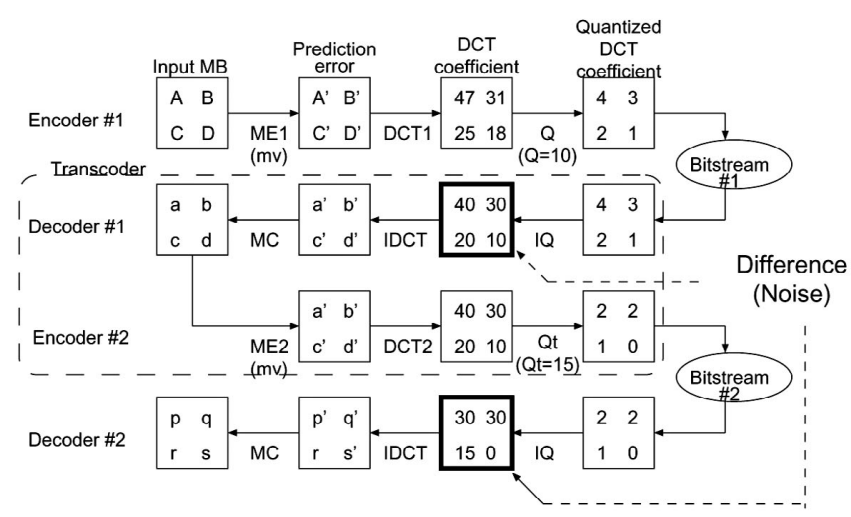

Fig. 4 Dataflow of CODEC chain $(\mathrm{Q}=10, \mathrm{Qt}=15)$.

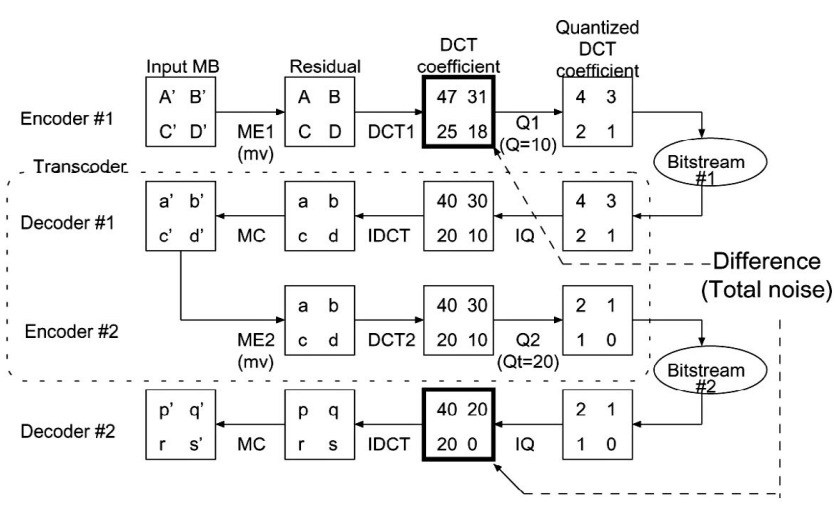

Fig. 5 Dataflow of CODEC chain $(Q=10, Q t=20)$. 


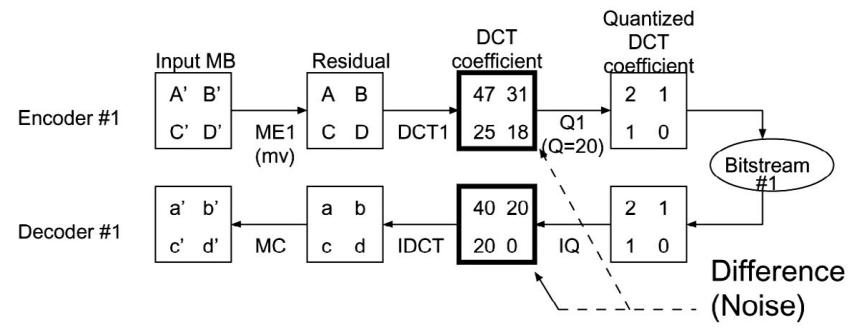

Fig. 6 Dataflow of CODEC $(\mathrm{Q}=20)$.

bitstream \#1 and generates bit stream \#2. The data flow of this CODEC chain is illustrated in Fig. 3. For simplification, in this figure we assume that (1) the macroblock size is $2 \times 2$, (2) the quantization and the de-quantization are done in accordance with Eq.1 and 2 with $\mathrm{p}=0,(3)$ the quantization parameter value is equal to its quantization step value, and (4) the value for all quantization matrix coefficients is sixteen. In the transcoder shown in the figure, the motion vector $m v$, the macroblock decision modes and the quantization parameter $Q$ are first extracted from bitstream \#1, and at the same time a decoded macroblock is generated by decoder \#1. Bitstream \#2 is obtained by reencoding the decoded macroblock using the old motion vector $m v$, the macroblock type, and the quantization parameter from bitstream \#1. The quantization noise Noise $_{t}$, which is mixed in the second re-quantization procedure, is expressed by the difference between the DCT coefficient of the first and second decoding procedures. In this example, there is no difference between the DCT coefficients because the old motion vector $m v$, the macroblock type, and the quantization parameter $Q$ are re-used in the second encoding procedure. This re-use makes all the procedures from the IDCT procedure in decoder $\# 1$ to the IQ procedure in decoder $\# 2$ reversible. The output bitstream \#2 is almost the same as the input bitstream \#1. This means there is no image quality degradation or bit reduction in the transcoder when the old motion vector, the macroblock type, and the quantization parameter are re-used. An example of a case where the encoder quantization parameters are different is shown in Fig. 4. In this example, the quantization parameter of the second encoder, $Q t$, is equal to 15 . This value is larger than the first encoder's quantization parameter, where $Q=10$. The size of bitstream \#2 is smaller than that of bitstream $\#$ 1. However, there is a clear difference between the DCT coefficients of the first and second decoders because the non-reuse of $Q$ makes all the procedures mentioned above un-reversible.
We will show that the noise level of the transcoding using $Q t=2 * Q$ is almost equals to the noise level of the encoding using $2 * Q$ in Fig. 5 and Fig. 6. Fig. 5 is a transcoding cxamplc of $Q=10$ and $Q t=20$. Fig. 6 is a encoding example of $Q=20$. The transcoder adds the quantization noise in two times. The first noise is the DCT coefficient difference between $(47,31,25,18)$ and $(40,30,20,10)$, and the second noise is the difference between $(40,30,20,10)$ and $(40,20,20,0)$. The total noise of the transcoder is the difference between $(47,31,25,18)$ and $(40,20,20,0)$. The encoding adds the quantization noise in once. It is the difference between $(47,31,25,18)$ and $(40,20,20,0)$. This indicates the noise level of the transcoding with $Q t=2 * Q$ is almost equals to those of the encoding with $2 * Q$.

\section{Previous work and problem}

A transcoding method called the RQMZT that avoids the image quality degradation described in the previous section has been reported ${ }^{7)}$. The basic idea of this method is to avoid the use of $Q t$, which degrades PSNR.

In RQMZT, some macroblocks are quantized by $Q t=$ $Q$, and the rest are quantized by $Q t=2 * Q$ instead of $Q t=1.5 * Q$, which would decrease PSNR for all macroblocks in the frame. When the percentage of the use of $Q t=2 * Q$ increases, the number of transcoding bits decreases. While the RQMZT method is superior to conventional methods in terms of PSNR, it has a serious problem. That is, in this method, bit reduction is achieved only by the macroblocks that are re-quantized by $Q t=2 * Q$. Those that are re-quantized by $Q t=Q$ produce no bit reduction. This bit reduction mechanism causes a difference in image quality between adjacent macroblocks that are quantized by $Q t=Q$ and those quantized by $Q t=2 * Q$. This problem does not degrade PSNR, but many people feel strange when they view such compressed images. We will show an example of this problem in Section 4.

\section{Proposed method}

To overcome the problem described in the previous section, we propose a transcoding method that reduces image quality degradation and minimizes image quality differences among macroblocks. We call it transcoding method based on a two-tiered quantization matrix (TTQM). Instead of changing the quantization parameter, as in conventional methods, the proposed method changes some elements of the quantization matrix. Quantization matrices $W I_{i, j}^{\prime}$ for intra-coding and 
$W N_{i, j}^{\prime}$ for non-intra-coding, shown in Eqs. (5) and (6), respectively, are used in this method.

$$
\begin{aligned}
& W I_{i, j}^{\prime}= \begin{cases}W I_{i, j} & \text { if }(i+j)<T H_{\text {intra }} \\
2 W I_{i, j}+1 & \text { otherwise }\end{cases} \\
& W N_{i, j}^{\prime}= \begin{cases}W N_{i, j} & \text { if }(i+j)<T H_{\text {inter }} \\
2 W N_{i, j} & \text { otherwise }\end{cases}
\end{aligned}
$$

where $W I$ is the quantization matrix that was used for intra-coding during the first encoding and $W N$ is the quantization matrix that was used for inter-coding during the first encoding. There is a dead zone in MPEG-2 intra-quantization, so $W I_{i, j}^{\prime}$ must be $2 W I_{i, j}+1$. The matrix elements that represent the high frequency part are doubled, while those for the lower frequency part are not altered. The $T H_{\text {inter }}$ and $T H_{\text {intra }}$ factors are thresholds located between the doubled elements and non-changed elements. They depend on the ratio of the transcoding bit rate to the first encoding bit rate. When a transcoding bit rate is almost the same as the first encoding bit rate, $T H_{\text {inter }}$ and $T H_{\text {intra }}$ are set to 14 or 15 . When the transcoding bit rate is slightly higher than half of the first encoding bit rate, they are set to 0 or 1. Doubling the value of the quantization matrix elements has almost the same effect as doubling the value of the quantization parameter. The only difference is that doubling the quantization parameter affects every frequency part of the image, but doubling the higher quantization elements only affects the higher frequency part. We mentioned in previous section that RQMZT can reduce re-quantization noise because the $Q t$ equals $Q$ or $2 * Q$. Our method can also reduce re-quantization noise because $Q t * W N_{i, j}^{\prime}$ equals $Q * W N_{i, j}$ or $Q * 2 W N_{i, j}$.

A quantization matrix example of the proposed method is shown in Fig. 7. It is for a flat quantization matrix in the first encoding procedure. Our method can also be applied to bitstreams compressed using a slant quantization matrix in the first encoder. It is well known that the enlargement of quantization matrix elements of the high frequency part improves encoding quality in video compression. On this basis, the MPEG2 default setting of the intra quantization matrix is slanted. Our proposed method also enlarges such elements. However there is a large difference between our method and such a simple slanting matrix method. Our method changes $W N_{i, j}$ to $2 W N_{i, j}$ instead of the value between $W N_{i, j}$ and $2 W N_{i, j}$, which would decrease the image quality for all macroblocks. However, such values between $W N_{i, j}$ and $2 W N_{i, j}$ are used in the slanting matrix method. The use of the values between $W N_{i, j}$ and $2 W N_{i, j}$ would increase the re-quantization noise in the slanting matrix method. The performance difference betwecn the proposed method and the other conventional methods, including the simple slanting matrix method, will be measured in Section 5 .

Fig. 8 illustrates the mechanism we used to compare the proposed method and three conventional methods (the slanting Q, the RQMZT, and the slanting matrix) in terms of bit-reduction and Q in macroblocks. Here, we assumed the original input image consisted of four macroblocks, the quantization parameter $Q=10$ was used in the first encoding, and each macroblock was represented by 20 bits. In the slanting $Q$ method, the slanting matrix method, and the proposed method, the bit count of each macroblock was reduced to 15 bits. This bit reduction was achieved in all macroblocks. However, in the RQMZT method, we obtained bit reduction in two macroblocks, \#1 and \#3, which wcre requantized by $Q t=20$. No bit reduction was achieved in macroblocks $\# 0$ and $\# 2$, which were re-quantized by $Q t=10$. There was no PSNR degradation in macroblocks \#0 and \#2, but \#1 and \#3 lost both the high and low frequency information included in the original image.

The image quality differences that cause feelings of strangeness are explained in Fig. 9 to Fig. 12. The simulation conditions and stream information are listed in Table 1. Fig. 9 is an enlargement of the original image. Fig. 10 is an enlarged picture compressed by the first encoder. Fig. 11 is an enlarged picture transcoded

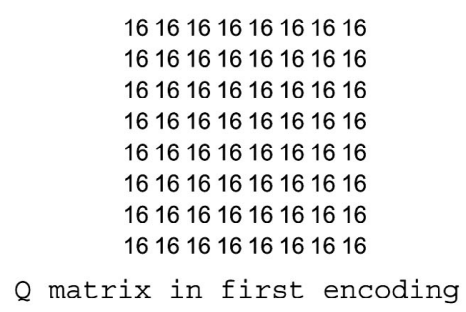

1616161616161616 1616161616161616 1616161616161616 $1616161616161616^{\bullet}$ 1616161616161616 1616161616161616 1616161616161616 1616161616161616

$\mathrm{TH}=15$ $1 6 1 6 1 6 1 6 1 6 \longdiv { 3 2 } 3 2 3 2$ $1 6 1 6 1 6 1 6 \longdiv { 3 2 3 2 3 2 3 2 }$ $1 6 1 6 1 6 \longdiv { 3 2 3 2 3 2 3 2 3 2 }$ 3232323232323232 $1616323232323232 \bullet \bullet 3232323232323232$

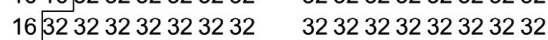
$3232323232323232 \quad 3232323232323232$ $3232323232323232 \quad 3232323232323232$ $3232323232323232 \quad 3232323232323232$

$\mathrm{Q}$ matrix for proposed TTQM method

Fig. 7 Quantization matrix examples of first encoding and proposed TTQM transcoding for inter macroblocks. 


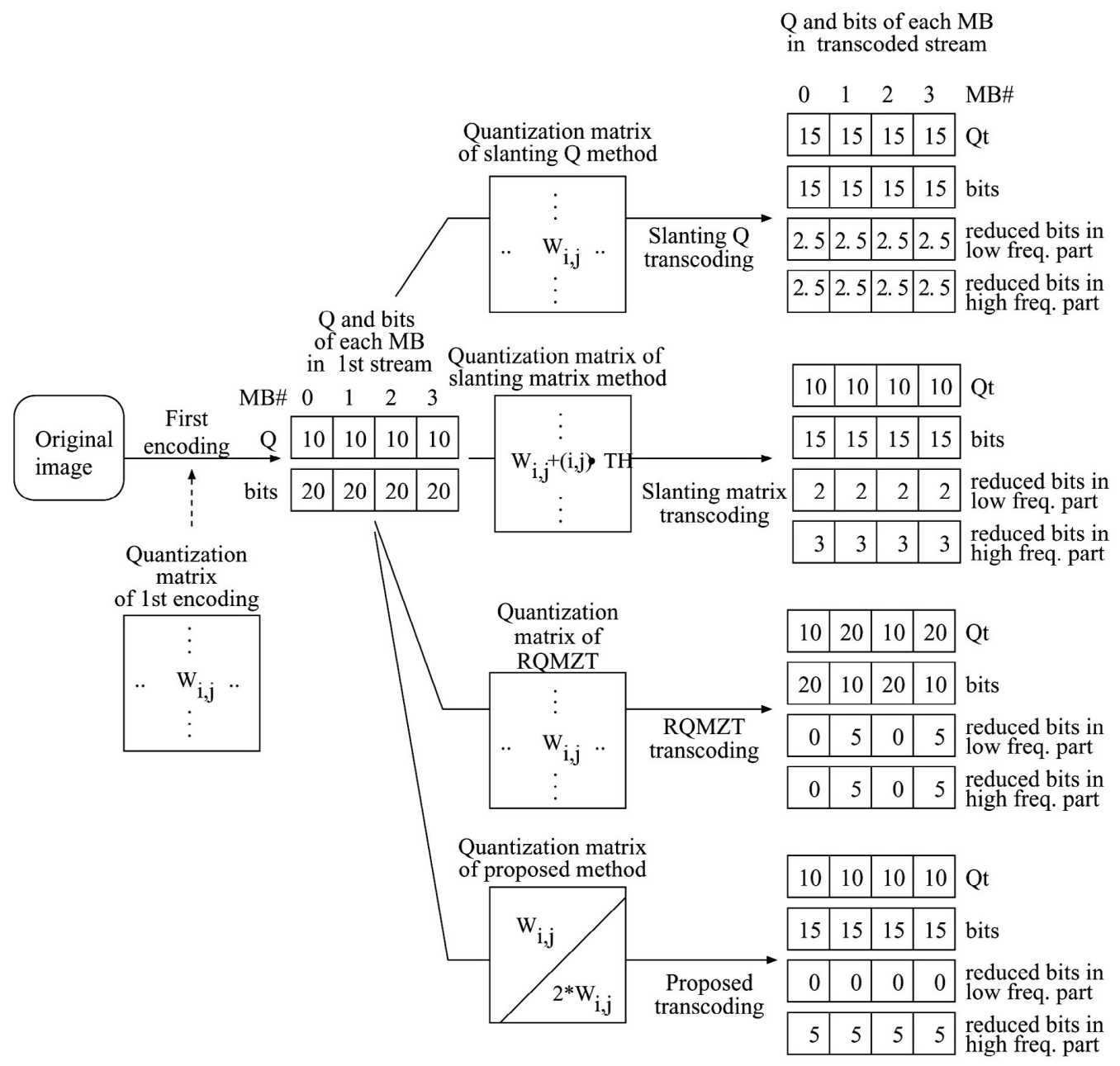

Fig. 8 Comparison of proposed method and three conventional methods in terms of bit-reduction and $Q$ in macroblocks.

by the RQMZT method. Fig. 12 is an enlarged picture transcoded by the proposed method. The size of these figures is $64 \times 64$ pixels, and sixteen macroblocks are included in each of the figures.

All macroblocks were quantized by $Q=24$ in the first encoding of the original image. The RQMZT transcoding and the proposed transcoding input the same decoded image compressed by the first encoder. In the

\begin{tabular}{c|c|c|c} 
Table 1 & \multicolumn{4}{c}{$\begin{array}{c}\text { Stream comparison of 1st encoding, transcod- } \\
\text { ing by RQMZT, and transcoding by proposed } \\
\text { method. }\end{array}$} \\
\hline \hline & $\begin{array}{c}\text { First } \\
\text { encoding }\end{array}$ & $\begin{array}{c}\text { Transcoding } \\
\text { by RQMZT }\end{array}$ & $\begin{array}{c}\text { Transcoding } \\
\text { by TTQM }\end{array}$ \\
\hline \hline Input image & $\begin{array}{c}\text { Mobile } \\
\text { and } \\
\text { calendar }\end{array}$ & $\begin{array}{c}\text { Decoded image } \\
\text { of first } \\
\text { encoding stream }\end{array}$ & $\leftarrow$ \\
\hline Image size & $720 \times 480$ & $\leftarrow$ & $\leftarrow$ \\
\hline Picture type & $\mathrm{I}$ & $\longleftarrow$ & $\leftarrow$ \\
\hline $\begin{array}{c}\text { Quantization } \\
\text { parameter }\end{array}$ & $\mathrm{Q}=24$ & $\mathrm{Qt}=24$ or 48 & $\mathrm{Qt}=24$ \\
\hline $\begin{array}{c}\text { Modification } \\
\text { of Q matrix }\end{array}$ & No & No & Yes \\
\hline $\begin{array}{c}\text { Output } \\
\text { stream }\end{array}$ & 57801 & 44971 & 44269 \\
size (bytes) & 28.257 & 25.175 & 24.522 \\
\hline PSNR (dB) & 0.897535 & 0.837288 & 0.839271 \\
\hline SSIM & $0.8510)$ \\
\hline VQM & 2.5588 & 3.4710 & 3.4125 \\
\hline $\begin{array}{c}\text { Enlargement } \\
\text { of decoded } \\
\text { image }\end{array}$ & Fig.10 & Fig.11 & Fig.12 \\
\hline
\end{tabular}

RQMZT method, the quantization matrix was not altered from that of the first encoder. One half of the macroblocks were then quantized by $Q t=24$, and the others were quantized by $Q t=48$. In the proposed method, the high frequency elements of the quantization matrix were doubled by using Eq. 5, after which all macroblocks were quantized by $Q t=24$. When the RQMZT method was used, the left eyes of the two animals in the image were clearer than when the proposed method was used. This was because the quantization parameter of the macroblocks representing the left eyes was the same as that of the first encoding. In contrast, the right eyes were blurrier than those obtained with the proposed method. There was a large quality difference between the macroblocks representing the left eyes and those representing the right eyes. This was because the quantization parameter of the macroblocks representing the right eyes was double that of the first encoding. This difference between the image quality of adjacent macroblocks degrades subjective image quality. Unfor- 

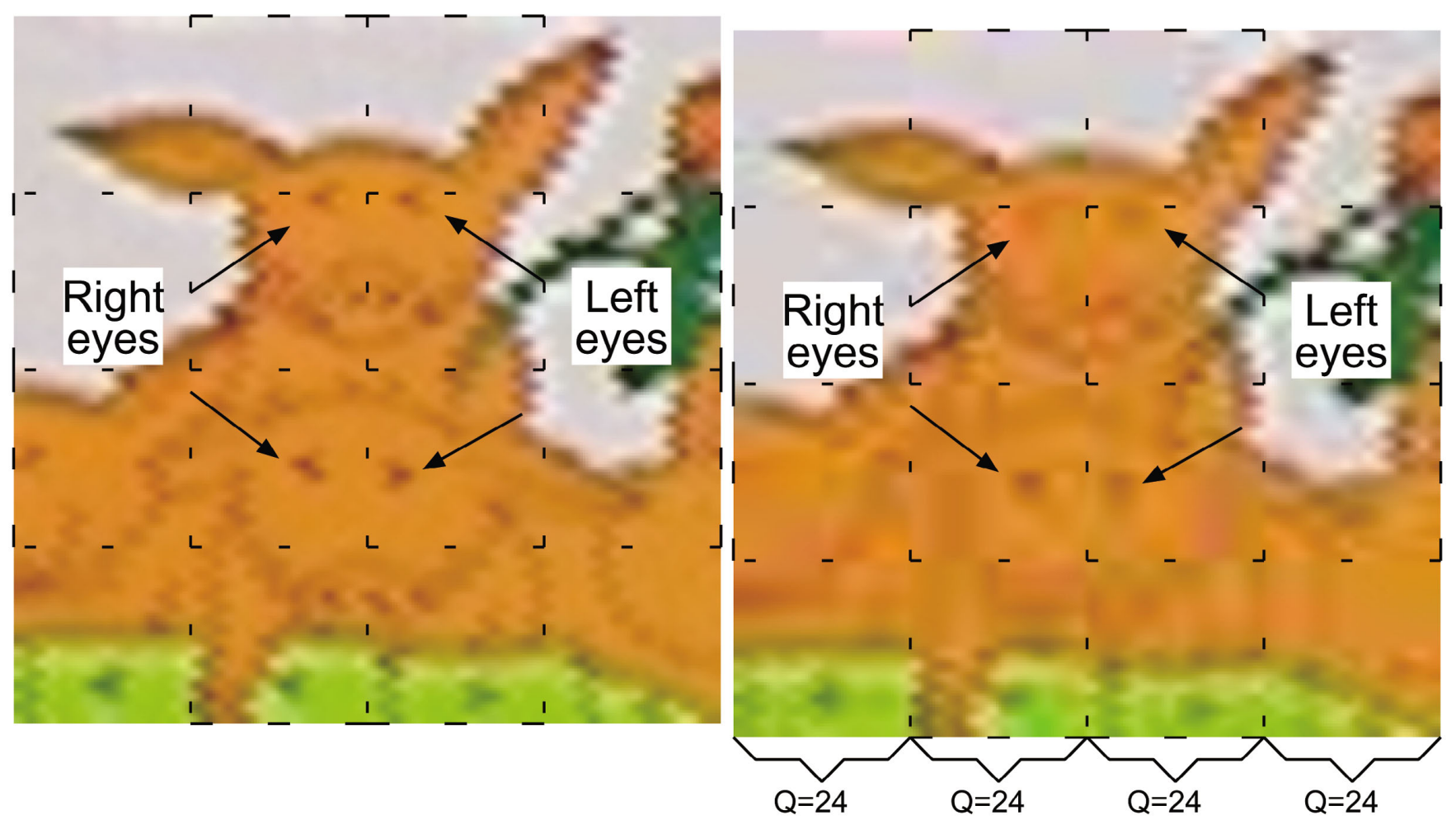

Fig. 9 Original image

Fig. 10 First encoded image.

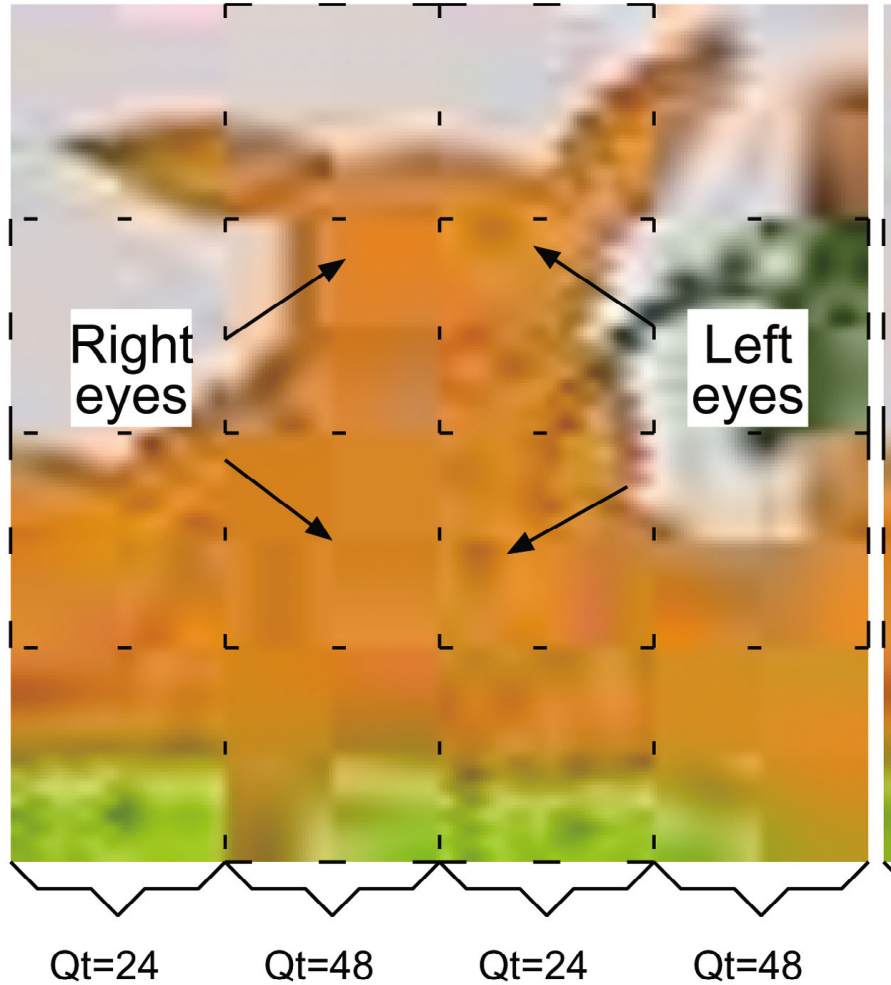

Fig. 11 Transcoded image by RQMZT method.

tunately, measuring this kind of degradation by using PSNR is difficult. The PSNR value is sometimes inconsistent with human visual perception because the noise is simply defined by the total mean squared error between the intensity of the input and the output pixels

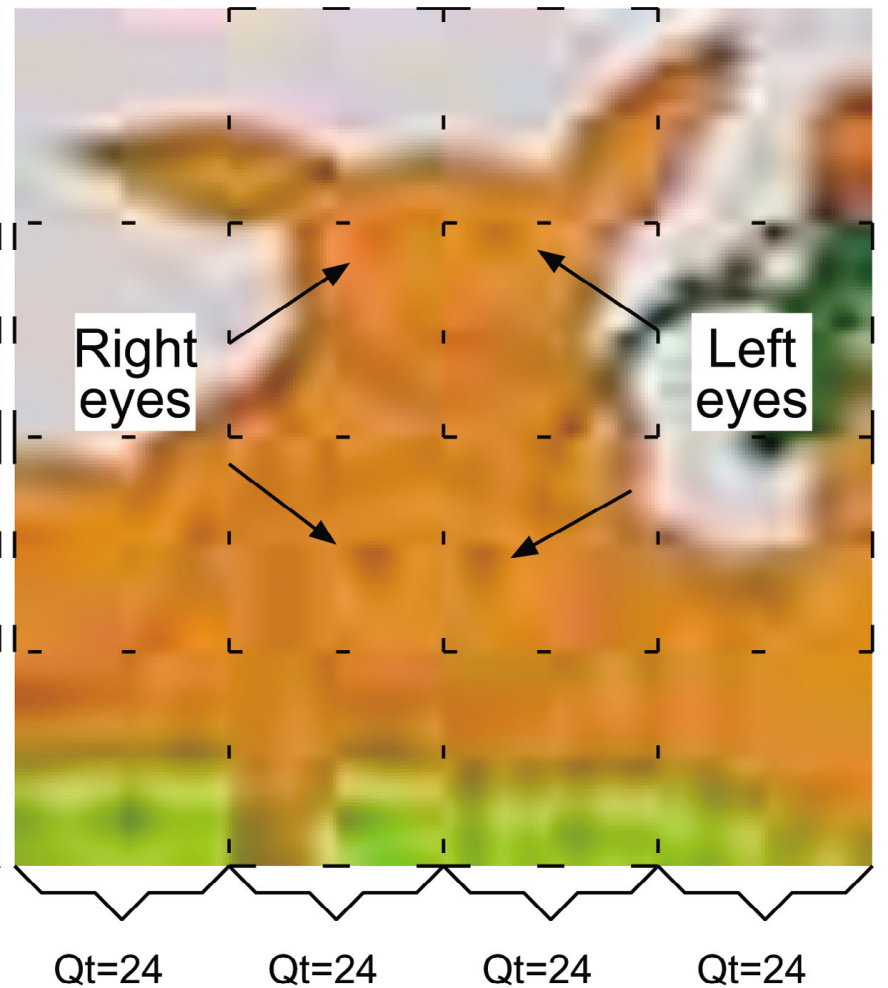

Fig. 12 Transcoded image by proposed method.

in the PSNR calculation.

The Video Quality Expert Group (VCEG), and ITUT have developed recommendations for objectively measuring video quality ${ }^{9)}$. We used the structural similarity $(\mathrm{SSIM})^{11) 12)}$ and the video qualily model $(\mathrm{VQM})^{10)}$ are 
used for the video quality measurements. The SSIM compares local patterns of pixel intensities that have been normalized for luminance and contrast. The SSIM index has becn shown to fit the mean option score (MOS) better than PSNR does in experimental measurements of 334 compressed scenes ${ }^{11)}$. SSIM is widely used as a quality evaluation standard for many compression methods. VQM is also closely correlated with the characteristics of the human visual systems and was standardized by ANSI in 2003. Please note that the VQM is different from PSNR and SSIM; increasing of the VQM value indicates that the video quality is worsening. The PSNR of our proposed method, shown in Table 1, was lower than that of RQMZT. However, the SSIM and VQM of our proposed method was better than that of RQMZT in that even the stream size of the proposed method was smaller than that of RQMZT.

\section{Experimental results}

In this section, we compare the performance of our proposed method with those of the conventional methods. To simulate our proposed method, we modified an MPEG-2 TM5 encoder to enable it to input all decisions made during the first encoding procedure. We prepared three conventional methods. The first one was RQMZT, which is described in Section 3. The second was a simple slanting matrix method, mentioned in Section 4. This method is similar to the proposed method in modifying the $\mathrm{Q}$ matrix, but it uses the $\mathrm{Q}$ matrix $W_{i, j}^{\prime}$, which is calculated by Eq. 7 instead of Eq 5, to enlarge the high frequency elements of the $\mathrm{Q}$ matrix. This means that the $\mathrm{Q}$ matrix $W_{i, j}^{\prime}$ increases in proportion to a rise in $i+j$.

$$
W_{i, j}^{\prime}=\min \left(W_{i, j}+(i+j) \cdot T H_{s l o p}, 255\right)
$$

$W_{i, j}^{\prime}$ equals $W_{i, j}$ when $T H_{\text {slop }}=0$. When $T H_{\text {slop }}$ increases, the slanting angle of the $W_{i, j}^{\prime}$ 's elements increases. Equation 7 is commonly used for both intraand inter-quantizaiton matrices. Quantization matrix examples of this simple slanting matrix method are shown in Fig. 13. The third method is a slanting Q method that enlarges the quantization parameter $\mathrm{Q}$ to decrease the generation of bits without modifying the Q matrix.

In our simulation, the first encoder used a fixed $Q$ value for each frame in a video sequence. The parameters for the first encoding and transcoding simulation are listed in Table 2 and Table 3. We used three video sequences $^{14)} 720 \times 480$ pixels in size for our compari- son. The input images of the proposed transcoder and these three conventional transcoders were obtained by MPEG-2 compression with a quantization parameter of $Q=16,24$, or 36 using an MPEG-2 default quantization matrix. The proposed transcoder and the slanting matrix encoder used the quantization parameter $Q t$, where $Q t=Q$. The former used a two-tiered quantization matrix that enabled the $T H$ to change from 0 to 15 , and the latter used a simple slanting matrix that enabled the $T H$ to change from 0 to 10 . The RQMZT transcoder and the slanting $\mathrm{Q}$ transcoder used the same $\mathrm{Q}$ matrix as that used in the first encoding. To decrease the generation of bits, these four transcoders took different approaches. The proposed transcoder chose a small $T H$ of the matrix modification, and the slanting matrix encoder chose a one. The RQMZT transcoder increased

Table 2 First encoding condition.

\begin{tabular}{c|l}
\hline \hline Sequence & Boat, Mobile and calendar, Plane \\
\hline $\mathrm{Q}$ & $\begin{array}{l}16 \text { or } 24 \text { or } 32 \\
\text { (fixed in a picture) }\end{array}$ \\
\hline $\begin{array}{c}\text { Q_scale_type } \\
\text { Quantization } \\
\text { matrix }\end{array}$ & $\begin{array}{l}\text { MPEG-2 default setting } \\
\text { (slanting matrix) }\end{array}$ \\
\hline GOP & IPPP $(\mathrm{N}=15)$ \\
\hline No. of frames & 30 \\
\hline
\end{tabular}

Table 3 Transcoding condition.

\begin{tabular}{|c|c|c|c|c|c|}
\hline \multirow{2}{*}{\multicolumn{2}{|c|}{$\begin{array}{l}\text { Transcoding } \\
\text { method }\end{array}$}} & \multirow{3}{*}{$\begin{array}{c}\text { Proposed } \\
\text { TTQM }\end{array}$} & \multicolumn{3}{|c|}{ Conventional method } \\
\hline & & & \multirow{2}{*}{$\begin{array}{l}\text { RQMZT } \\
16 \text { or } 32\end{array}$} & \multirow{2}{*}{$\begin{array}{c}\begin{array}{c}\text { Slanting } \\
\text { matrix }\end{array} \\
16\end{array}$} & \multirow{2}{*}{$\begin{array}{c}\text { Slanting } \\
Q \\
16,18 . ., 32\end{array}$} \\
\hline \multirow{3}{*}{ Qt } & $\mathrm{Q}=16$ & & & & \\
\hline & $\mathrm{Q}=24$ & 24 & 24 or 48 & 24 & $24,26 . ., 48$ \\
\hline & $\mathrm{Q}=32$ & 32 & 32 or 60 & 32 & $32,34 \ldots, 60$ \\
\hline \multicolumn{2}{|c|}{$\begin{array}{c}\text { Quantization } \\
\text { matrix }\end{array}$} & $\begin{array}{l}\text { Modificd } \\
\text { by Eq. } 5 \\
\text { or Eq. } 6\end{array}$ & $\begin{array}{l}\text { Default } \\
\text { setting }\end{array}$ & $\begin{array}{l}\text { Modificd } \\
\text { by Eq. } 7\end{array}$ & $\begin{array}{l}\text { Default } \\
\text { setting }\end{array}$ \\
\hline \multicolumn{2}{|r|}{ TH } & 0 to 15 & - & 0 to 10 & - \\
\hline \multicolumn{2}{|c|}{ Re-use } & $\mathrm{Q}, \mathrm{mv}$ & $\mathrm{mv}$ & $\mathrm{Q}, \mathrm{mv}$ & $\mathrm{mv}$ \\
\hline
\end{tabular}

1616161616161616 1616161616161616 1616161616161616 1616161616161616 1616161616161616 1616161616161616 1616161616161616 1616161616161616

Q matrix in first encoding

$1616161616161616 \quad 1617181920212223 \quad 1618202224262830$ $1616161616161616 \quad 1718192021222324 \quad 1820222426283032$ $1616161616161616 \quad 1819202122232425 \quad 2022242628303234$ $1616161616161616 \quad 1920212223242526 \quad 2224262830323436$ $1616161616161616 \quad 2021222324252627 \quad 2426283032343638$ $1616161616161616 \quad 2122232425262728 \quad 2628303234363840$ $1616161616161616 \quad 2223242526272829 \quad 2830323436384042$ $1616161616161616 \quad 2223242526272829 \quad 3032343638404244$

$$
\mathrm{TH}=0 \quad \mathrm{TH}=1 \quad \mathrm{TH}=2
$$$$
\text { Q matrix for slanting matrix method }
$$

Fig. 13 Quantization matrix examples of first encoding and slanting matrix transcoding. 

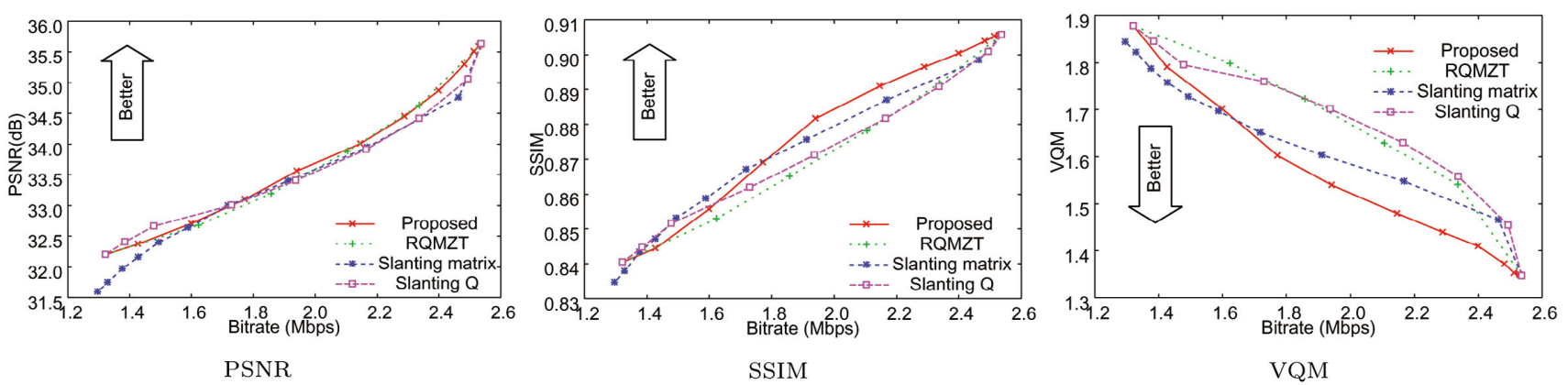

Fig. 14 Relationship between (PSNR, SSIM, VQM) and bitrate for "Boat" compressed by first encoder using $\mathrm{Q}=16$.
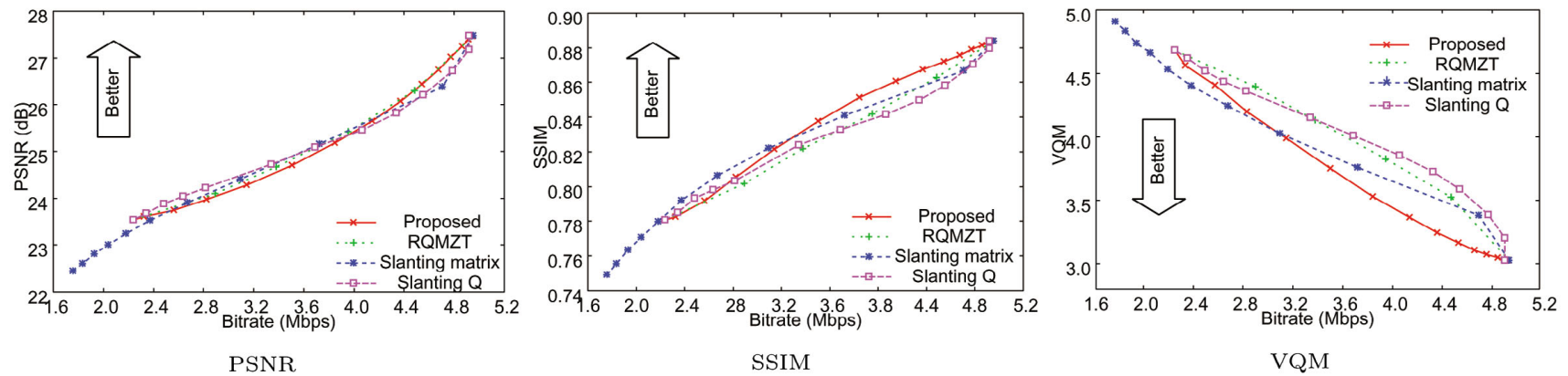

Fig. 15 Relationship between (PSNR, SSIM, VQM) and bitrate for "Mobile and calendar" compressed by first encoder using $\mathrm{Q}=24$.
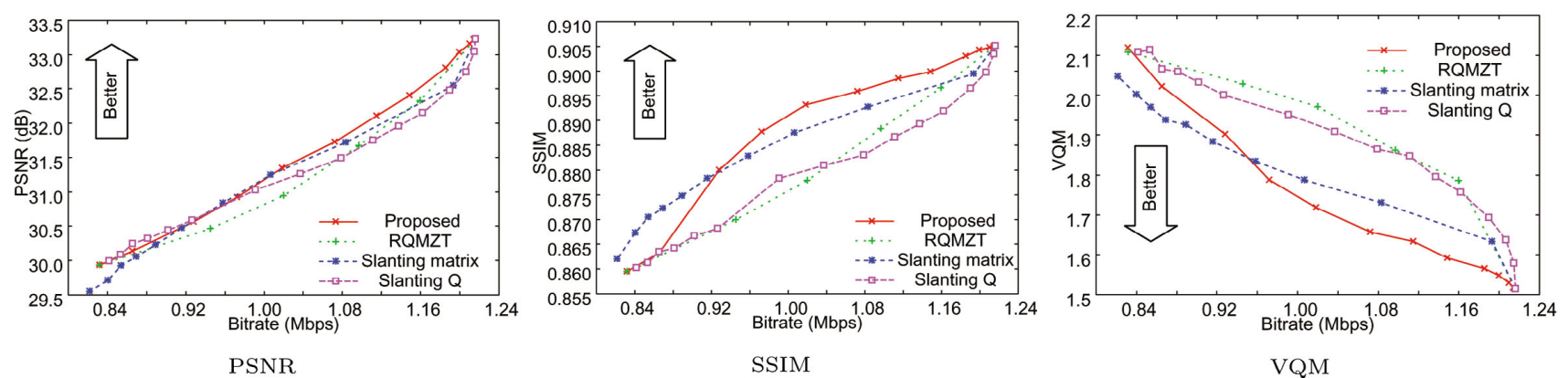

Fig. 16 Relationship between (PSNR, SSIM, VQM) and bitrate for "Plane" compressed by first encoder using $\mathrm{Q}=32$

the frequency of using $Q t=2 Q$, and decreased the frequency of using $Q t=Q$ to decrease the generation of bits. The slanting $\mathrm{Q}$ transcoder selected a large $Q t$, where $Q t>Q$.

The relationship between the three evaluation scores (PSNR, SSIM, and VQM) and the bitrate for the three tested sequences are shown in Fig. 14 to Fig. 16. The input sequence was compressed by the first encoder, where $\mathrm{Q}=16$. Concerning the PSNR results, the proposed method and the RQMZT were superior to other methods when the transcoding bit rate was slightly lower than the first encoding bit rate. When the transcoding bit rate was slightly higher than half of the first encoding bit rate, the slanting-Q method was superior to other methods. In terms of SSIM and VQM re- sults, the proposed method was superior to other methods, especially when the amount of bit reduction was small. As the transcoding bit rate became smaller, the advantage of the proposed method decreased with regard to SSIM and VQM.

We compared our proposed transcoding method with conventional methods using three evaluation scores when the bit reduction ratio was 0.2 . The simulation results are listed in Table 4 . The score improvements obtained with the proposed method vis-a-vis that of the best results obtained by the conventional methods are shown in the right-hand "improvement in proposed method" column. Since we reversed the sign of the VQM improvement score in this column, positive values mean that the proposed method is superior to the 
Table 4 Video quality scores of proposed method and three conventional methods when bit reduction ratio $=0.2$

\begin{tabular}{|c|c|c|c|c|c|c|c|c|}
\hline \multirow{2}{*}{\multicolumn{4}{|c|}{$\begin{array}{c}\text { Input } \\
\text { (First encoding result) }\end{array}$}} & \multirow[b]{2}{*}{$\begin{array}{l}\text { Proposed } \\
\text { method }\end{array}$} & \multicolumn{3}{|c|}{ Conventional methods } & \multirow{2}{*}{$\begin{array}{l}\text { Improvement } \\
\text { in proposed } \\
\text { method }\end{array}$} \\
\hline & & & & & RQMZT & $\begin{array}{l}\text { Slanting } \\
\text { Matrix }\end{array}$ & $\begin{array}{c}\text { Slanting } \\
\text { Q }\end{array}$ & \\
\hline \multirow{3}{*}{ Scene } & & Bitrate & PSNR & PSNR & PSNR & PSNR & PSNR & PSNR \\
\hline & $\mathrm{Q}$ & [kbps] & SSIM & SSIM & SSIM & SSIM & SSIM & SSIM \\
\hline & & & VQM & VQM & VQM & VQM & VQM & VQM \\
\hline \multirow{9}{*}{ Boat } & & & 35.6288 & 33.7494 & 33.6698 & 33.6477 & 33.6097 & 0.0796 \\
\hline & 16 & 2536 & 0.9057 & 0.8856 & 0.8742 & 0.8808 & 0.8754 & 0.0048 \\
\hline & & & 1.3468 & 1.5139 & 1.6574 & 1.5773 & 1.6724 & 0.0634 \\
\hline & & & 33.7743 & 32.0325 & 31.7889 & 31.8491 & 31.7046 & 0.1833 \\
\hline & 24 & 1691 & 0.8747 & 0.8503 & 0.8338 & 0.8455 & 0.8376 & 0.0047 \\
\hline & & & 1.5945 & 1.7863 & 1.9812 & 1.8570 & 1.9950 & 0.0707 \\
\hline & & & 32.5717 & 30.7050 & 30.4234 & 30.6537 & 30.5002 & 0.0513 \\
\hline & 32 & 1335 & 0.8511 & 0.8155 & 0.7990 & 0.8172 & 0.8092 & -0.0018 \\
\hline & & & 1.5945 & 2.0300 & 2.2234 & 2.0633 & 2.2281 & 0.0333 \\
\hline \multirow{9}{*}{$\begin{array}{l}\text { Mobile } \\
\text { and } \\
\text { calendar }\end{array}$} & & & 30.0456 & 27.2195 & 27.5851 & 27.5472 & 27.6184 & -0.3989 \\
\hline & 16 & 7796 & 0.9204 & 0.8919 & 0.8866 & 0.8870 & 0.8832 & 0.0049 \\
\hline & & & 2.2916 & 2.7907 & 2.9894 & 2.9195 & 3.0462 & 0.1288 \\
\hline & & & 27.4701 & 25.3610 & 25.4351 & 25.4537 & 25.3596 & -0.0927 \\
\hline & 24 & 4953 & 0.8839 & 0.8549 & 0.8424 & 0.8473 & 0.8393 & 0.0077 \\
\hline & & & 3.0264 & 3.4650 & 3.8189 & 3.6675 & 3.8972 & 0.2025 \\
\hline & & & 25.8666 & 24.1975 & 24.0667 & 24.1439 & 23.9872 & 0.0537 \\
\hline & 32 & 3554 & 0.8522 & 0.8238 & 0.8033 & 0.8150 & 0.8042 & 0.0088 \\
\hline & & & 3.0264 & 3.9939 & 4.4429 & 4.2305 & 4.5312 & 0.2367 \\
\hline \multirow{9}{*}{ Plane } & & & 36.3857 & 34.1192 & 34.1292 & 34.2277 & 34.2466 & -0.1274 \\
\hline & 16 & 2159 & 0.9323 & 0.9186 & 0.9116 & 0.9154 & 0.9084 & 0.0033 \\
\hline & & & 1.1249 & 1.3147 & 1.4591 & 1.3475 & 1.4322 & 0.0328 \\
\hline & & & 34.4949 & 32.2901 & 32.0606 & 32.3392 & 32.2763 & -0.0491 \\
\hline & 24 & 1503 & 0.9165 & 0.9025 & 0.8905 & 0.8985 & 0.8900 & 0.0039 \\
\hline & & & 1.3454 & 1.5678 & 1.7777 & 1.6112 & 1.7283 & 0.0434 \\
\hline & & & 33.2281 & 30.9312 & 30.6390 & 30.9653 & 30.9042 & -0.0341 \\
\hline & 32 & 1216 & 0.9051 & 0.8878 & 0.8729 & 0.8843 & 0.8754 & 0.0036 \\
\hline & & & 1.3454 & 1.7869 & 2.0071 & 1.8201 & 1.9650 & 0.0333 \\
\hline \multirow{3}{*}{\multicolumn{2}{|c|}{ Average }} & & 32.1667 & 30.0672 & 29.9775 & 30.0919 & 30.0229 & -0.0371 \\
\hline & & 2971 & 0.8935 & 0.8701 & 0.8571 & 0.8656 & 0.8580 & 0.0044 \\
\hline & & & 1.8551 & 2.2499 & 2.4841 & 2.3437 & 2.4995 & 0.0938 \\
\hline
\end{tabular}

other methods in every evaluation. The average SSIM and VQM scores of the proposed method were found to be about 0.0044 and 0.0938 better, respectively, than those of the best scoring conventional method for the tested video sequences.

We calculated the upper limit by which the proposed method was superior to the best scoring conventional method in terms of SSIM and VQM for several input bitstreams. The results are shown in Table 5. The "Bit reduction ratio" column means the ratio of the bitstream reduction size to the input bitstream size in this table. They indicate that the proposed method was superior to the best scoring conventional method when the bit reduction ratio was smaller than 0.2531 at the lower limit average of the SSIM score. In the VQM score, the proposed method was superior when the bit reduction ratio was smaller than 0.3211 . This also indicates that the proposed method was always superior in terms of both SSIM and VQM scores when the bit reduction ratio was smaller than 0.1015 for all the tested sequences.

\section{Conclusion}

An MPEG-2 transcoding method using a two-tiered quantization matrix that reduces re-quantization noise is proposed. Instead of changing the value of the quan-
Table 5 Upper limit of bit reduction ratio at which proposed TTQM method was superior to best of other methods.

\begin{tabular}{|c|c|c|c|c|c|c|}
\hline \multirow{3}{*}{ Scene } & \multirow{3}{*}{$\mathrm{Q}$} & \multirow{3}{*}{$\begin{array}{l}\text { Input } \\
\text { stream } \\
\text { bitrate } \\
\text { (kbps) }\end{array}$} & \multicolumn{4}{|c|}{ Upper limit of TTQM dominated } \\
\hline & & & \multicolumn{2}{|c|}{ SSIM } & \multicolumn{2}{|c|}{ VQM } \\
\hline & & & $\begin{array}{c}\text { Bitrate } \\
\text { (kbps) }\end{array}$ & $\begin{array}{l}\text { Bit } \\
\text { reduction } \\
\text { ratio }\end{array}$ & $\begin{array}{c}\text { Bitrate } \\
\text { (kbps) }\end{array}$ & $\begin{array}{l}\text { Bit } \\
\text { reduction } \\
\text { ratio }\end{array}$ \\
\hline \multirow{4}{*}{ Boat } & 8 & 5160 & 3550 & 0.3120 & 3220 & 0.3760 \\
\hline & 16 & 2536 & 1786 & 0.2957 & 1634 & 0.3557 \\
\hline & 24 & 1691 & 1281 & 0.2425 & 1167 & 0.3099 \\
\hline & 32 & 1335 & 1084 & 0.1880 & 1009 & 0.2442 \\
\hline \multirow{4}{*}{$\begin{array}{l}\text { Mobile } \\
\text { and } \\
\text { calendar }\end{array}$} & 8 & 15578 & 11719 & 0.2477 & 12258 & 0.2131 \\
\hline & 16 & 7796 & 5398 & 0.3076 & 5102 & 0.3456 \\
\hline & 24 & 4953 & 3284 & 0.3370 & 3072 & 0.3798 \\
\hline & 32 & 3554 & 2444 & 0.3123 & 2188 & 0.3844 \\
\hline \multirow{4}{*}{ Plane } & 8 & 4256 & 3824 & 0.1015 & 2732 & 0.3581 \\
\hline & 16 & 2159 & 1412 & 0.3460 & 1505 & 0.3029 \\
\hline & 24 & 1503 & 1081 & 0.2808 & 1095 & 0.2715 \\
\hline & 32 & 1216 & 925 & 0.2393 & 951 & 0.2179 \\
\hline \multirow{4}{*}{$\begin{array}{l}\text { Cheer } \\
\text { leader }\end{array}$} & 8 & 12814 & 10320 & 0.1946 & 9536 & 0.2558 \\
\hline & 16 & 6568 & 5041 & 0.2325 & 4624 & 0.2960 \\
\hline & 24 & 4274 & 3314 & 0.2246 & 3036 & 0.2897 \\
\hline & 32 & 3240 & 2544 & 0.2148 & 2328 & 0.2815 \\
\hline \multirow{4}{*}{ Race } & 8 & 5908 & 3526 & 0.4032 & 3226 & 0.4540 \\
\hline & 16 & 2319 & 1617 & 0.3027 & 1356 & 0.4153 \\
\hline & 24 & 1364 & 1113 & 0.1840 & 924 & 0.3226 \\
\hline & 32 & 1018 & 876 & 0.1395 & 718 & 0.2947 \\
\hline \multirow{4}{*}{ Show } & 8 & 9374 & 8099 & 0.1360 & 6064 & 0.3531 \\
\hline & 16 & 4733 & 3342 & 0.2939 & 3072 & 0.3509 \\
\hline & 24 & 3107 & 2214 & 0.2874 & 2066 & 0.3350 \\
\hline & 32 & 2366 & 1770 & 0.2519 & 1654 & 0.3009 \\
\hline \multicolumn{2}{|c|}{ Average } & 4534 & 3398 & 0.2531 & 3105 & 0.3211 \\
\hline
\end{tabular}

tization parameters as conventional methods do, our transcoding method modifies the higher frequency elements of the quantization matrix to avoid losing image information for the lower frequency parts in the input image. Experimental results show that our proposed method produces transcoding SSIM and VQM scores 
that are about 0.0044 and 0.0938 better, respectively, than those of the best conventional method without causing any difference in image quality between adjacent macroblocks. When the bit reduction ratio was smaller than 0.1015 , the proposed method was always superior to the best conventional method.

\section{〔References〕}

1) G. Keesman, R. Hellinghuizen, F. Hoeksema, and G. Heideman, "Transcoding of MPEG bitstream" Signal Processing: Image Communication, 8, pp.481-500 (1996)

2) H. Sun, W. Kwok, and J.W. Zdepski, "Architectures for MPEG compressed bitstream scaling" IEEE Trans. Circuit \& Syst. for Video Technology, 6, 2, pp.191-199 (April 1996)

3) P. Assuncao and M. Ghanbari, "Post-processing of coded video for transmission at lower bit rates" Proc. IEEE Int. Conf. ASSP, 4, pp.1998-2001 (1996)

4) W. Kou and T.Fjallbrant, "A direct computation of DCT coefficients for a signal taken from two adjacent blocks" IEEE Trans. Signal Processing, 39,7, pp.1692-1695 (July 1991)

5) P.Guilotel, et al, "Adaptive Encoders: The New Generation of MPEG-2 Encoders" SMPTE journal (April 2000)

6) S.Kadono, M.Etho, and N.Yokoya, "Rationality of restricted requantization for efficient MPEG transcoding " Proc. 2000 International Conference on Image Processing : 952-5, 1 (2000)

7) S.Kadono, M.Etho, N.Yokoya "Re-Quantization Method with Zone Thresholding for MPEG Transcoding" IEICE Transaction J83-D-11,9, pp.1879-1887 (November 1997)

8) S.Kadono, M.Etho, and N.Yokoya, "Optimization of ReQuantization Parameter for MPEG Transcoding" IEICE Transaction J84-D-II, 7, pp.1389-1398 (July 2001)

9) Final report from the video quality experts group on the validation of objective models of video quality assessment, phase II (August 2003)

10) Pinson, M., and Wolf, S., A new standardized method for objectively measuring video quality" IEEE Trans. Broadcast., 2004, 50, pp.312-322

11) Z. Wang, L. Lu, and A. C. Bovik, Video quality assessment based on structural distortion measurement, Signal Processing: Image Communication, special issue on objective video quality metrics, 19, 2, pp. 121-132 (Feb. 2004)

12) Z. Wang and E. P. Simoncelli, Translation insensitive image similarity in complex wavele $t$ domain, IEEE International Conference on Acoustics, Speech and Signal Processing, II, pp. 573-576 , Philadelphia, PA (Mar. 2005)

13) MPEG-2, Test Model 5 (TM5), Doc ISO/IEC JTC/SC29/WG11/N0400, Test Model Editing Commitee (April 1993)

14) ITU-R Recommendation BT.802, "Test pictures and sequences for subjective assessments of digital codec conveying signals produced according to recommendation ITU-R BT.601” (1994)

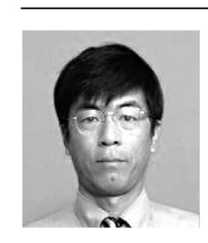

Takeshi Yoshitome received the B.S. and M.S. degrees in computer science from Tsukuba University, Japan, in 1982 and 1984. In 1984, he joined the Electrical Communication Laboratories, Nippon Telegraph and Telephone Corporation (NTT), Kanagawa, Japan, where he since been engaged in research and development of image processing systems. He is currently a Senior Research Engineer of the Visual Media Communications Project in NTT Cyber Space Laboratories, Kanagawa, Japan.

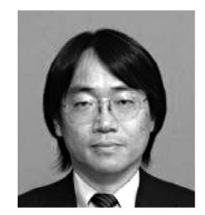

Jiro Naganuma received the B.S. degree in electrical engineering from Tokushima University, Tokushima, Japan in 1981, and the Ph.D. degree in information science from Kyoto University, Kyoto, Japan in 1995. In 1981, he joined Musashino Electrical Communication Laboratories, Nippon Telegraph and Telephone Corporation (NTT), Tokyo, Japan. He is currently a senior engineer for the NTT Electronics Corporation. He is engaged in the research of high-level VLSI architectures and design methodologies for multimedia embedded system LSIs. Dr Naganuma is a member of the Information Processing Society of Japan (IPSJ), and the IEEE Computer Society.

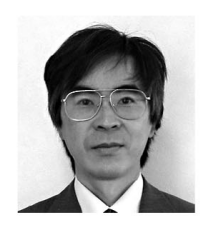

Yoshiyuki Yashima received the B.E., M.E., and Ph.D degrees from Nagoya University, Nagoya, Japan, in 1981, 1983, and 1998 respectively. In 1983 he joined the Electrical Communications Laboratories, Nippon Telegraph and Telephone Corporation (NTT), Kanagawa, Japan, where he has been engaged in the research and development of high quality HDTV signal compression, MPEG video coding algorithms, and lossless image coding systems. His research interests also include pre- and post-processing for video coding, processing of compressed video, compressed video quality metrics and image analysis for video communication systems. He has been a visiting professor of Tokyo Institute of Technology since 2004. $\mathrm{He}$ is currently a professor of Information and Network Science at Chiba Institute of Technology. He was awarded the Takayanagi Memorial Technology Prize in 2005. Dr.Yashima is a member of the IEEE Signal Processing Society, the Information Processing Society of Japan (IPSJ), the Institute of Electronics, Information, and Communication Engineers of Japan (IEICE), and the Institute of Image Information and Television Engineers of Japan (ITE). 